\title{
DESEMPENHO EM PROVA DE VOCABULÁRIO DE CRIANÇAS COM DESVIO FONOLÓGICO E COM DESENVOLVIMENTO FONOLÓGICO NORMAL
}

\section{Performance in vocabulary test of children with phonological disorder and with typical phonological developmental}

\author{
Ana Rita Brancalioni (1), Caroline Marini (2), Laura Giotto Cavalheiro (3), Márcia Keske-Soares ${ }^{(4)}$
}

\begin{abstract}
RESUMO
Objetivo: comparar o desempenho em prova específica de vocabulário de crianças com e sem desvio fonológico. Método: participaram da pesquisa 150 crianças, 75 do Grupo com Desvio Fonológico (GDF) e 75 do Grupo com Desenvolvimento Fonológico Normal (GDFN), entre 6:0 e 6:11, de ambos os gêneros, pertencentes ao nível socioeconômico médio. Realizaram-se as triagens fonoaudiológica e auditiva, a Avaliação Fonológica da Criança e a avaliação do Vocabulário. As respostas das crianças foram analisadas considerando o número de Designações Verbais Usuais (DVU), Não Designações (ND) e Processos de Substituição (PS), por campo conceitual. Posteriormente, os dados foram tabulados e submetidos à análise estatística através do Teste $T$ para amostras independentes, considerando-se $p<0,05$. Resultados: verificou-se que o GDF obteve menor número de DVU que o GDFN, sendo esta diferença estatisticamente significante para todos os campos conceituais. Além disso, a ocorrência de PS e ND foi maior para o GDF, em todos os campos conceituais, havendo diferença estatisticamente significante respectivamente, para oito e quatro campos conceituais. Ainda, a ocorrência de PS foi maior que a de ND para ambos os grupos. Conclusão: o GDF apresentou desempenho inferior na prova do vocabulário, verificado pela menor ocorrência de DVU e maior de ND e PS que o GDFN. Esses achados mostram haver relação entre desvio fonológico e déficit no vocabulário. Assim, é importante avaliar o vocabulário das crianças com desvio fonológico para auxiliar na terapia.
\end{abstract}

DESCRITORES: Vocabulário; Patologia da Fala e Linguagem; Semântica; Criança

(1) Fonoaudióloga; Bolsista do CAPES; Mestranda em Distúrbios da Comunicação Humana na Universidade Federal de Santa Maria.

(2) Fonoaudióloga; Clínica Otorrinos Reunidos, COR, Caxias do Sul, RS, Brasil; Mestranda em Distúrbios da Comunicação Humana na Universidade Federal de Santa Maria.

(3) Fonoaudióloga; Professora dos Cursos de Fonoaudiologia das Faculdades Jorge Amado, FJA, Salvador, BA, Brasil; da Faculdade Nobre de Feira de Santana, FAN, Feira de Santana, BA, Brasil; e da União Metropolitana de Educação e Cultura, UNIME, Lauro de Freitas, BA, Brasil; Mestre em Distúrbios da Comunicação Humana pela Universidade Federal de Santa Maria.

(4) Fonoaudióloga; Professora do Curso de Fonoaudiologia e do Programa de Pós-graduação em Distúrbios da Comunicação Humana da Universidade Federal de Santa Maria, UFSM, Santa Maria, RS, Brasil; Bolsista do Conselho Nacional de Desenvolvimento Científico e Tecnológico, CNPq-Brasil; Doutora em Linguística Aplicada pela Pontifícia Universidade Católica do Rio Grande do Sul.

Conflito de interesses: inexistente

\section{INTRODUÇÃO}

Toda criança ao longo do desenvolvimento e experiência constrói um léxico mental, que é acessado à medida que deseja representar, por meio de palavras, um objeto, uma ação, um acontecimento. Aprender palavras e saber utilizá-las adequadamente é um aspecto fundamental do desenvolvimento da linguagem e está relacionado à aquisição da sintaxe, da morfologia e da fonologia ${ }^{1,2}$.

Estudos ${ }^{3-6}$ revelam que crianças com dificuldades no aspecto fonológico também apresentam déficit no aspecto semântico. Contudo, outros estudos ${ }^{1,7}$ sugerem que uma dificuldade no nível fonológico não interfere significativamente no desempenho do vocabulário.

A aquisição lexical requer o estabelecimento de uma correspondência entre a forma fonológica de 
uma palavra e sua representação semântica ${ }^{8}$. As categorias de maior produção no desenvolvimento inicial do vocabulário são, respectivamente, substantivos, verbos, adjetivos e advérbios ${ }^{9,10}$. A frequência, a familiaridade ${ }^{4,8,11-13}$, e a extensão das palavras ${ }^{14}$, além do estímulos ambientais ${ }^{15}$, são fatores que parecem interferir na aquisição do léxico.

A recepção verbal e o atraso da expressão são indicativos de desenvolvimento tardio do uso da linguagem e apresenta forte correlação com baixo índice de inteligência e déficit nas habilidades acadêmicas ${ }^{16}$. Quando se observa falta de correspondência entre o significado da palavra da linguagem adulta e o significado dessa mesma palavra na linguagem da criança tem-se um desvio ${ }^{2}$.

Estudo ${ }^{17}$ sugere que o vocabulário e a memória fonológica estão relacionados desde o início do desenvolvimento da linguagem. Além disso, a dificuldade da aprendizagem do vocabulário pode ser devido à compreensão limitada da atenção e da audição, ou da memória fonológica de curto prazo. Tal dificuldade pode ser atribuída a dificuldades específicas na habilidade da criança armazenar o padrão da palavra dentro de sua memória ou apresentar pobres representações semânticas que limitam o desenvolvimento de um vocabulário mais amplo ${ }^{18}$.

O desvio fonológico é caracterizado por uma desorganização, inadaptação ou anormalidade no sistema de sons da criança em relação ao padrão de sua comunidade linguística, estando ausentes quaisquer comprometimentos orgânicos, como deficiência auditiva, comprometimento neurológico e anormalidades anatômicas ou fisiológicas nos mecanismos de produção da fala. Além disso, a criança deve apresentar idade superior a quatro anos, com linguagem expressiva adequada em termos de vocabulário e extensão do enunciado ${ }^{19}$. Contudo, algumas dessas características clínicas podem ser revistas, como o desempenho do vocabulário, que em alguns casos de desvio fonológico, encontra-se abaixo do esperado ${ }^{3-6}$. Um estudo ${ }^{20}$ sugere que a gravidade do desvio fonológico, pode influenciar o desempenho do vocabulário.

Diante disso, a presente pesquisa teve o objetivo de comparar o desempenho de crianças com desvio fonológico, com idades entre 6:0 e 6:11, em prova específica de vocabulário ${ }^{21} \mathrm{com}$ a performance de crianças da mesma faixa etária com desenvolvimento fonológico normal.

\section{MÉTODO}

Esta pesquisa é de caráter transversal, e do tipo quantitativa. A amostra foi composta por 150 crianças, com idades entre 6:0 e 6:11, de ambos os gêneros e de nível socioeconômico médio. Para classificação do nível socioeconômico foi realizado estudo estatístico baseado no Índice de Desenvolvimento Sócio-Econômico - IDESE ${ }^{22}$, considerando os indicadores de desenvolvimento apresentados no Anuário da cidade de Salvador/BA de 2003.

Inicialmente, todas as crianças foram submetidas à anamnese, triagem fonoaudiológica e triagem auditiva. Foram excluídas da amostra as crianças que apresentaram alterações quanto à linguagem, motricidade orofacial que pudessem interferir na fala, comprometimento auditivo e sinais indicativos de alterações neurológicas e psicológicas.

Como critério de inclusão as crianças deveriam apresentar audição normal; não apresentar problemas neurológicos relevantes à produção da fala; não apresentar distúrbio articulatório; ter capacidades intelectuais adequadas para o desenvolvimento da linguagem; ter adequada compreensão da linguagem para a faixa etária; apresentar desenvolvimento fonológico normal ou apresentar desvio fonológico.

As crianças foram submetidas às avaliações fonológica, a qual foi realizada através do instrumento da Avaliação Fonológica da Criança (AFC) ${ }^{23}$ e de vocabulário, em que foi utilizada a Avaliação do Vocabulário do Teste de Linguagem Infantil nas áreas de fonologia, vocabulário, fluência e pragmática (ABFW) ${ }^{21}$.

A avaliação fonológica ${ }^{23}$ foi realizada através da nomeação de cinco figuras temáticas ("zoológico", "sala", "cozinha", "banheiro" e "veículos") permitiu a obtenção de uma amostra espontânea de fala envolvendo todos os fones contrastivos em todas as posições que ocorrem na sílaba e na palavra. Os dados coletados foram gravados em ambiente silencioso, através do gravador digital da marca PalmOne modelo Zire 72, com microfone acoplado da marca PIONNER, modelo DM-30. Realizou-se a transcrição fonética ampla que, posteriormente, foi conferida por dois juízes. Por fim, foi realizada análise contrastiva com finalidade de estabelecer o inventário fonético e o sistema fonológico da criança, para comparar com o alvo adulto.

A partir dos resultados das avaliações fonológica e de vocabulário, a amostra foi dividida em dois grupos: Grupo com Desvio Fonológico (GDF), composto por 75 crianças e Grupo com Desenvolvimento Fonológico Normal (GDFN), composto, também, por 75 crianças. Ambos os grupos não foram pareados quanto à variável sexo, uma vez que em estudo realizado ${ }^{10}$ não se observou diferença estatística quanto ao sexo no desempenho do vocabulário.

A classificação do GDF foi determinada conforme os critérios de um estudo ${ }^{24}$ em que a criança 
apresenta fala com desvios em relação à pronúncia-alvo adulta, demonstrando compreensão adequada a sua idade mental, capacidade intelectual adequada para o desenvolvimento da linguagem $e$ audição normal. Ainda, não apresentando anormalidade anatômica ou fisiológica do mecanismo de produção da fala e/ou disfunção neurológica.

A avaliação do vocabulário ${ }^{21}$ possibilitou verificar a competência lexical e os mecanismos utilizados pelas crianças, no que se refere à quantidade de vocábulo - Designações por Vocábulos Usuais (DVU), Não Designações (ND) e Processos de Substituição (PS) - utilizados em lugar da palavra alvo. Esse instrumento é composto por nove campos conceituais (Vestuário; Animais; Alimentos; Meios de Transporte; Móveis e Utensílios; Profissões; Locais; Formas e Cores; Brinquedos e Instrumentos Musicais), totalizando o reconhecimento e a nomeação de 118 figuras. A avaliação e análise dos resultados do teste foram realizadas conforme instruções determinadas pela autora.

Para coleta dos dados foram necessárias, três sessões individuais, em média de 40 minutos, para cada criança. A primeira destinada à triagem fonoaudiológica e avaliação fonológica, a segunda à triagem audiológica e a última à avaliação do vocabulário.

Esta pesquisa foi aprovada pelo Comitê de Ética em Pesquisa sob número 0013.0.243.000-06, da Comissão Nacional de Ética e Pesquisa (CONEP). Ressalta-se que os diretores das escolas assinaram o Termo de Autorização Institucional e os pais e/ou responsáveis pelas crianças, assinaram o Termo de Consentimento Livre e Esclarecido, autorizando a participação das mesmas na pesquisa.

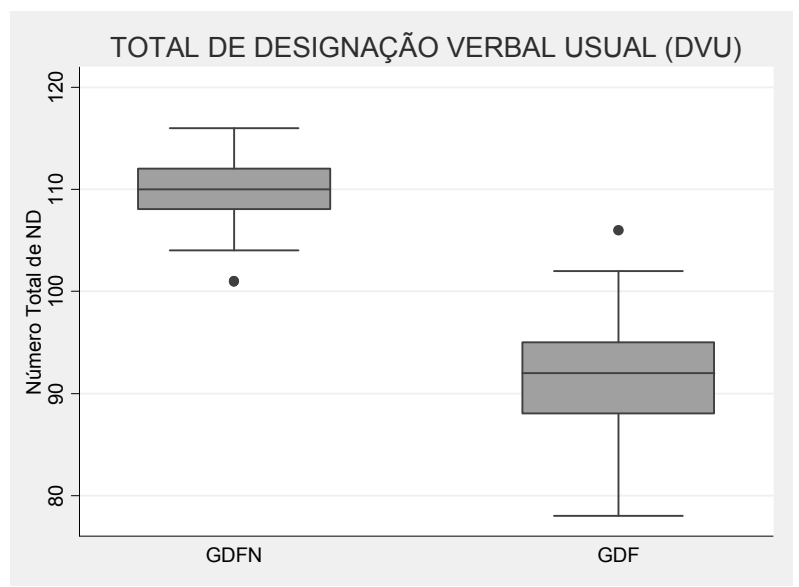

Análise estatística utilizando test $t$, obteve $p=0,0001$

Figura 1 - Box-Plot para o número total de Designação Verbal Usual para o Grupo com Desenvolvimento Fonológico Normal (GDFN) e para o Grupo com Desvio Fonológico (GDF)
As respostas das crianças foram analisadas individualmente, considerando o número de ocorrências de Designações por Vocábulos Usuais (DVU), Não Designações (ND) e Processos de Substituição (PS), por campo conceitual. Posteriormente, os dados foram tabulados e submetidos à análise estatística por meio do Teste T para amostras independentes, considerando-se $\mathrm{p}<0,05$.

\section{RESULTADOS}

Na Figura 1 é apresentado o gráfico Box-Plot do número total de ocorrência de Designações por Vocábulos Usuais (DVU) para GDFN e GDF. Observa-se que o valor da mediana, representada pela linha no interior do Box, foi menor para o GDF (92) do que para o GDFN (110). Considerando os valores mínimos e máximos de Designações por Vocábulos Usuais (DVU), observa-se que o GDF obteve valores inferiores (78 e 102) ao GDFN (106 e 116). Diante disso, observa-se que o GDF apresentou menor ocorrência de Designações por Vocábulos Usuais (DVU) quando comparada com o GDFN e, a análise estatística, utilizando o Teste T para amostras iguais, revelou diferença estatisticamente significante $(p=0,0001)$.

Nota-se a presença de valores de discrepância para o GDFN, na qual somente uma criança apresentou 101 ocorrências de DVU, e para o GDF, na qual apenas uma criança obteve 106 ocorrências de DVU.

A Figura 2 mostra o gráfico Box-Plot do número total de ocorrência de Não Designações (ND) para o GDFN e o GDF. Verifica-se que $75 \%$ das crianças

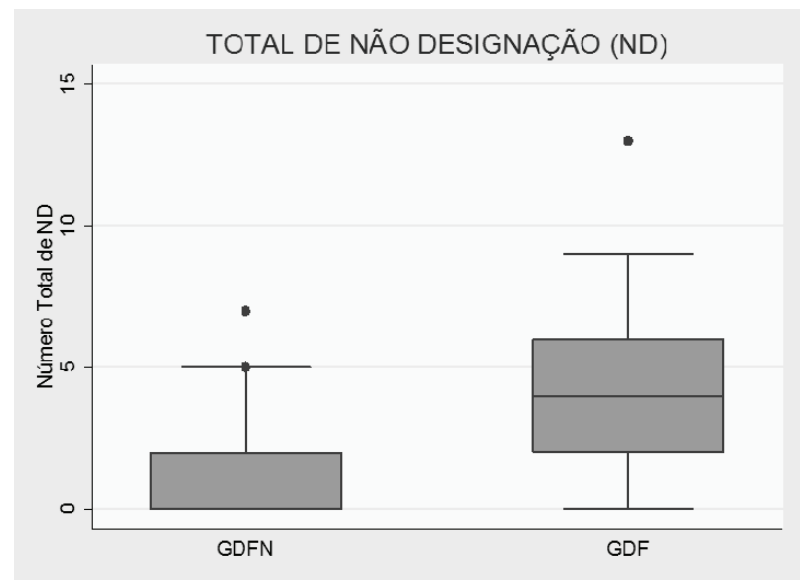

Análise estatística, utilizando Test $t$, obteve $p=0,0003$

Figura 2 - Box-Plot para o número total de Não Designação para o Grupo com Desenvolvimento Fonológico Normal (GDFN) e para o Grupo com Desvio Fonológico (GDF) 
do GDFN apresentaram duas ou menos ocorrências de Não Designações (ND) e que a mediana para este grupo foi de nenhuma ocorrência. Considerando o GDF, em geral, as crianças deste grupo apresentaram maior ocorrência de Não Designações (ND) quando comparadas as do GDFN. Ainda, a análise estatística resultou em $p=0,0003$, sendo encontrados três valores de discrepância.

A Figura 3 ilustra o Box-Plot para o número total de Processos de Substituição (PS) para GDFN e GDF. É possível observar que os valores da mediana, mínimo-máximo, primeiro e terceiro quartiis foram maiores para o GDF. Importante ressaltar que a análise estatística mostrou haver diferença estatística entre os grupos $(p=0,0004)$. Nenhum valor de discrepância foi encontrado.

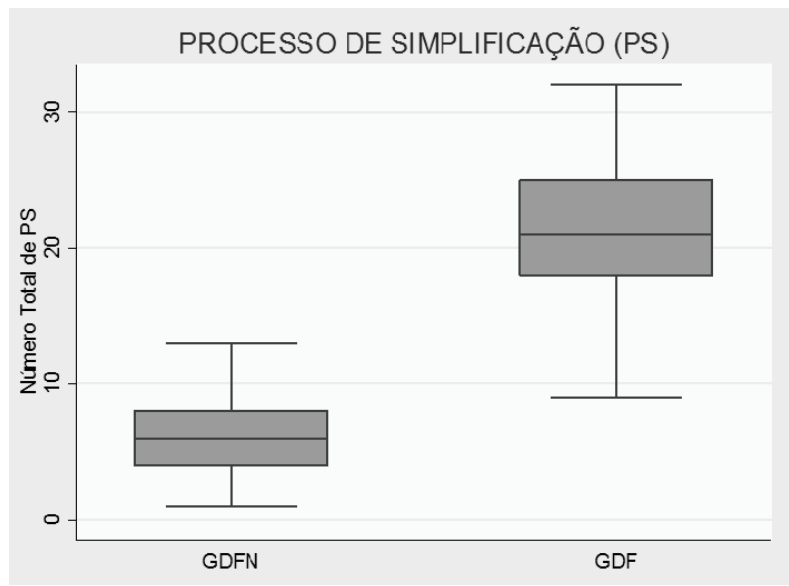

Análise estatística, utilizando Test $t$, obteve $p=0,0002$

Figura 3 - Box-Plot para o número total de Processos de Simplificação para o Grupo com Desenvolvimento Fonológico Normal (GDFN) e para o Grupo com Desvio Fonológico (GDF)
A Figura 4 mostra a média dos percentuais de Designações por Vocábulos Usuais (DVU), por campo conceitual, para GDFN e GDF. Observa-se que o GDF obteve menores percentuais de Designações por Vocábulos Usuais (DVU) em todos os campos conceituais.

$\mathrm{Na}$ Figura 5 é ilustrada a média dos percentuais de Não Designações (ND), por campo conceitual, para GDFN e GDF. Maiores percentuais de Não Designações (ND) foram apresentados pelo GDF em todos os campos conceituais.

A Figura 6 representa a média dos percentuais de Processos de Substituição (PS), por campo conceitual, para GDFN e GDF. Verifica-se que o GDF obteve maiores percentuais de Processos de Substituição em todos os campos conceituais.

A Tabela 1 apresenta a média dos percentuais de Designações por Vocábulos Usuais (DVU), Não Designações (ND) e Processos de Substituição (PS) por campo conceitual, bem como o desvio padrão, para GDFN e GDF. Quanto a Designações por Vocábulos Usuais (DVU), as crianças do GDF tiveram desempenho inferior as do GDFN, em todos os campos conceituais, sendo esta diferença estatisticamente significante.

As crianças do GDF não designaram um maior número de possibilidades de nomeação, para todos os campos conceituais, sendo esta diferença estatisticamente significante para quatro campos conceituais: Vestuário, Alimentos, Meios de Transporte e Móveis e Utensílios.

Referente à ocorrência de Processos de Substituição, esta foi maior para o GDF, em todos os campos conceituais, não havendo diferença estatisticamente significante apenas para o campo conceitual Formas e Cores.

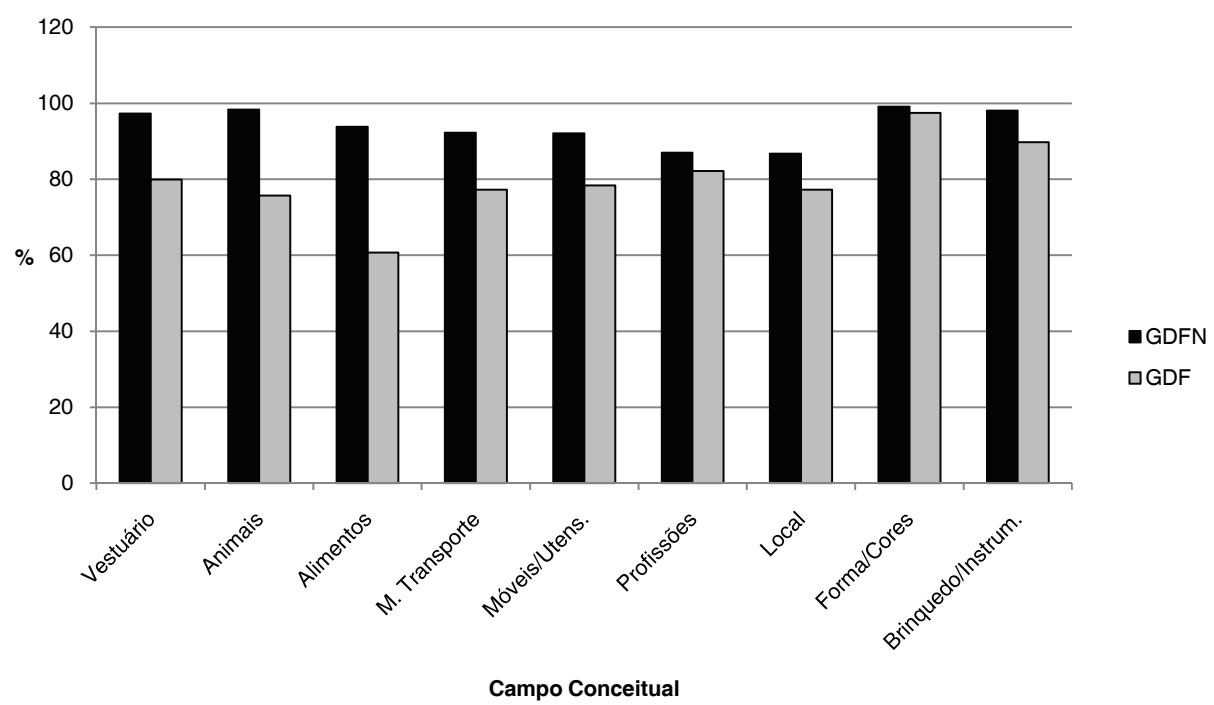

Figura 4 - Média dos percentuais de DVU por cmapo conceitual, para os GDFN e GDF 


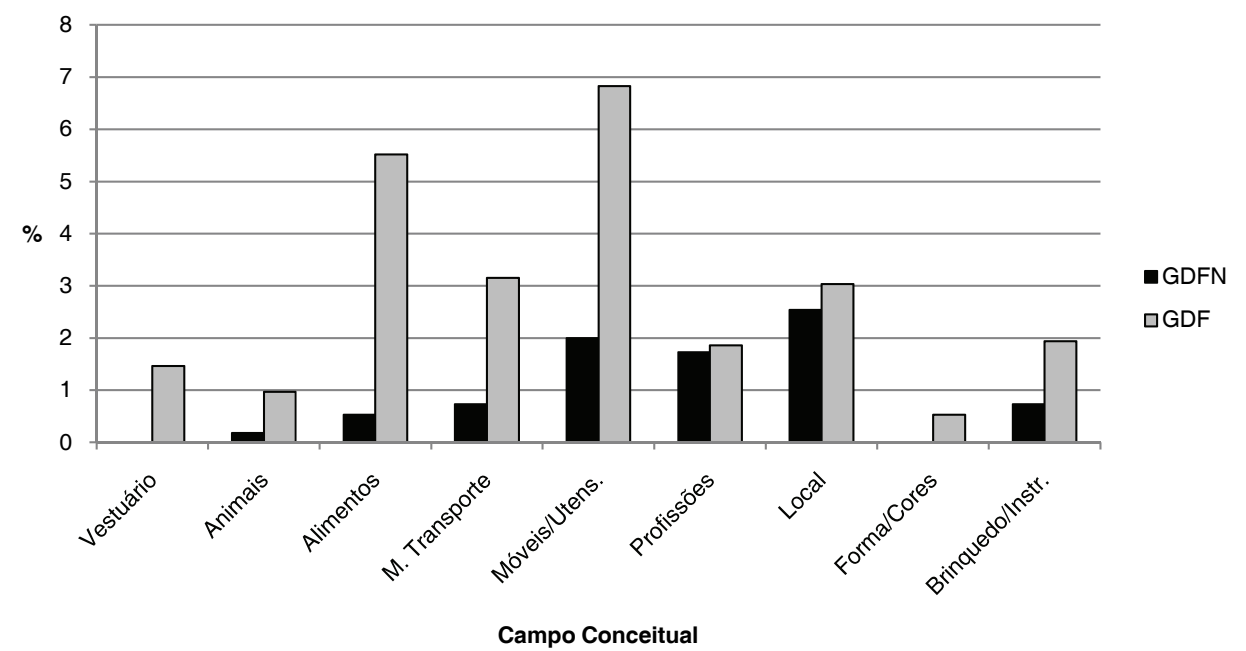

Figura 5 - Média dos percentuais de ND por cmapo conceitual, para os GDFN e GDF

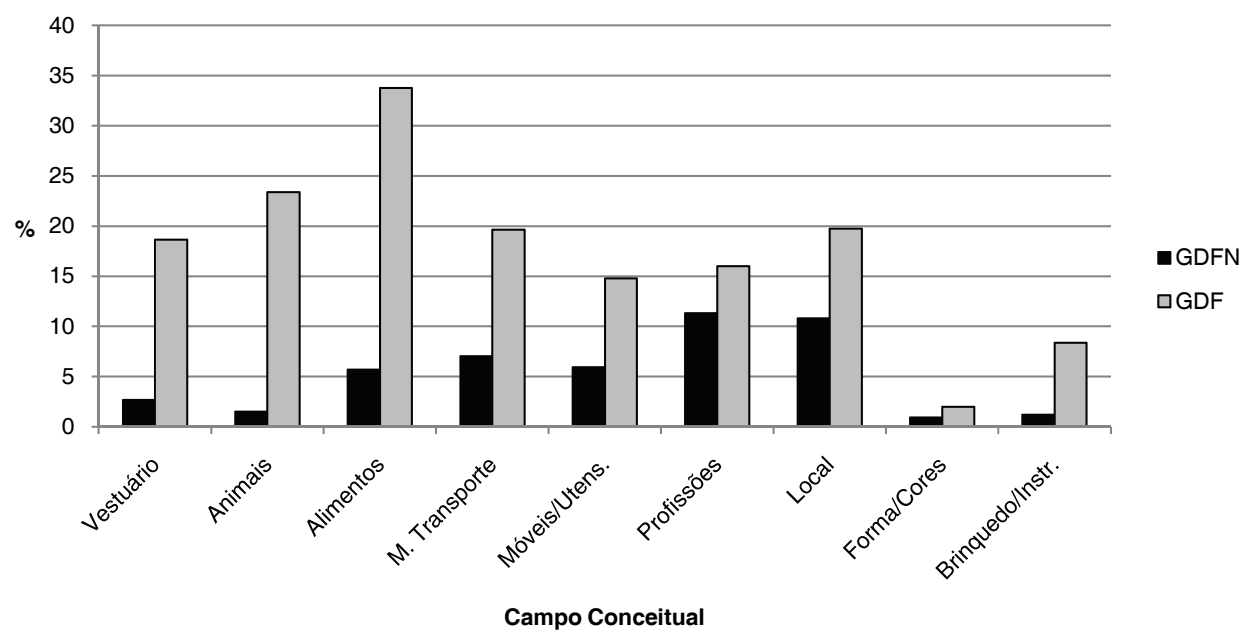

Figura 6 - Média dos percentuais de ND por campo conceitual, para os GDFN e GDF

\section{DISCUSSÃO}

Os achados desta pesquisa concordam com a literatura ${ }^{3-6}$, a qual refere haver uma estreita relação entre fonologia e léxico, pois se verificou que o GDF apresentou desempenho inferior ao GDFN na prova específica do vocabulário. Contudo, esses resultados discordam dos achados de outros estudos ${ }^{1,7}$, os quais revelaram que as crianças com alteração fonológica apresentaram vocabulário semelhante ao de crianças com desenvolvimento fonológico normal, utilizando o mesmo instrumento de avaliação.

Entre os fatores que influenciam a aquisição do vocabulário, estudo ${ }^{15}$ revela que os estímulos oferecidos pela mãe à criança são importantes na aquisição de novas palavras. Há uma correlação positiva entre o estímulo linguístico dos pais e a produção verbal da criança. Assim, através da experiência e do contexto, a criança aprimora sua capacidade de aprender o significado de uma nova palavra e também de organizar a informação obtida no convívio social e físico, por meio da interação com o adulto ${ }^{4,8}$.

As características do modo de fala dos pais e a habilidade de categorizar são fatores determinantes para a composição do vocabulário da criança e para a aquisição de formas linguísticas mais complexas ${ }^{4}$. Ressalta-se nessa pesquisa que, embora o nível de estimulação dos pais possa ter sido diferenciado entre as crianças, todas (GDFN e GDF) faziam parte do mesmo nível sócio-econômico (médio), cujos pais eram alfabetizados e não apresentavam alteração de linguagem.

Outro fator que pode interferir na aquisição do vocabulário é a idade da criança ${ }^{1,2,4,10,20,25}$ na qual 
Tabela 1 - Média de percentual de DVU, ND e OS, por cmapo conceitual para o GDNF e GDF

\begin{tabular}{lcccc}
\hline CATEGORIA & POSSIBILIDADE & GDFN & GDF & p \\
\hline \multirow{3}{*}{ Vestuário } & DVU & $97,33(6,00)$ & $79,88(10,96)$ & 0,0000 \\
& ND & $0,00(0,000)$ & $1,46(4,84)$ & 0,0053 \\
Animais & PS & $2,67(6,00)$ & $18,66(10,56)$ & 0,0000 \\
& DVU & $98,31(0,594)$ & $75,66(2,708)$ & 0,0000 \\
Alimentos & ND & $0,18(0,162)$ & $0,97(0,671)$ & 0,0682 \\
& PS & $1,51(0,582)$ & $23,37(2,616)$ & 0,0000 \\
\hline \multirow{3}{*}{ Meio de Transporte } & DVU & $93,78(1,638)$ & $60,71(2,037)$ & 0,0000 \\
& ND & $0,53(0,394)$ & $5,52(1,480)$ & 0,0002 \\
& PS & $5,69(1,616)$ & $33,77(2,256)$ & 0,0000 \\
\hline \multirow{3}{*}{ Móveis e Utensílios } & DVU & $92,24(0,691)$ & $77,21(1,473)$ & 0,0000 \\
& ND & $0,73(0,273)$ & $3,15(0,762)$ & 0,0027 \\
& PS & $7,03(0,669)$ & $19,64(1,470)$ & 0,0000 \\
\hline \multirow{2}{*}{ Profissões } & DVU & $92,06(2,179)$ & $78,39(2,644)$ & 0,0000 \\
& ND & $2,00(1,234)$ & $6,83(2,044)$ & 0,0000 \\
& PS & $5,94(1,415)$ & $14,78(2,139)$ & 0,0000 \\
\hline \multirow{2}{*}{ Locais } & DVU & $86,94(1,207)$ & $82,14(1,193)$ & 0,0030 \\
& ND & $1,73(0,644)$ & $1,86(0,747)$ & 0,4535 \\
Formas e Cores & PS & $11,33(1,017)$ & $16,00(1,078)$ & 0,0036 \\
\hline \multirow{2}{*}{ Instrumentos } & DVU & $86,67(1,233)$ & $77,22(1,178)$ & 0,0000 \\
\hline & ND & $2,54(0,648)$ & $3,03(0,664)$ & 0,3098 \\
& PS & $10,79(1,086)$ & $19,75(1,329)$ & 0,0000 \\
\hline & DVU & $99,07(0,408)$ & $97,47(0,659)$ & 0,0383 \\
& ND & $0,00(0,000)$ & $0,53(0,279)$ & 0,0514 \\
& PS & $0,93(0,408)$ & $2,00(0,677)$ & 0,1227 \\
\hline & DVU & $98,06(0,663)$ & $89,70(1,244)$ & 0,0000 \\
& ND & $0,73(0,358)$ & $1,94(0,642)$ & 0,0597 \\
& PS & $1,21(0,474)$ & $8,36(1,010)$ & 0,0000 \\
\hline
\end{tabular}

o léxico torna-se mas complexo com o avanço da idade ${ }^{26}$. No entanto, todas as crianças desta pesquisa encontravam-se na mesma faixa etária, revelando que este aspecto não interferiu no desempenho das provas realizadas, não justificando, consequentemente, a diferença dos resultados entre os grupos (GDFN e GDF).

Como observado na Figura 1, o GDF obteve um resultado inferior ao GDFN na nomeação de palavras em Designações por Vocábulos Usuais (DVU). Isso corrobora outro estudo ${ }^{3}$, o qual refere que um déficit na aquisição fonológica gera dificuldades em vários níveis da linguagem, como, por exemplo, erros em relação aos contrastes fonológicos, uso de processos fonológicos não esperados para a idade, e alterações no léxico.

Além de ter apresentado menos Designações por Vocábulos Usuais (DVU), verificou-se que o GDF apresentou maior número de Não Designações (ND) e Processos de Substituição (PS) que o GDFN, o que pode refletir a experiência reduzida em recuperar elementos lexicais ${ }^{11,12}$.

Houve, ainda, maior ocorrência de Processos de Substituição (PS) que Não Designações (ND), tanto no GDF quanto no GDFN. Isso mostra que é mais comum a criança realizar um processo de substituição do que não nomear um objeto. Esse achado concorda com os resultados de outros estudos ${ }^{4,11}$, os quais verificaram que as crianças tendem a produzir substituições que são mais frequentes, aprendidas mais cedo e que são mais próximas, em densidade e frequência do que a palavra-alvo.

A nomeação inclui um processo adicional de identificação caracterizada por três estágios: identificação do objeto, ativação conhecida, e geração da resposta ${ }^{27}$. Verifica-se que a menor ocorrência de Não Designações (ND) quando comparada com Processos de Substituição (PS), revela que as crianças de ambos os grupos passaram pelos estágios de identificação, contudo podem ter 
apresentado dificuldades nesses aspectos. Porém, mesmo assim, é mais comum a nomeação do objeto por uso de processos do que a ausência de respostas. Corroborando outro estudo ${ }^{6}$, que sugere que a ocorrência de substituições de palavras por outras, com atributos semânticos próximos, pode indicar que a criança com desvio fonológico conhece o objeto, porém é incapaz de recuperar a palavra.

Quanto às Designações por Vocábulos Usuais (DVU), a média do percentual foi inferior para o GDF, em todos os campos conceituais avaliados, havendo, para todos, diferença estatisticamente significante. Isso porque, as dificuldades em nomeação, nas crianças com dificuldades fonológicas, devem-se à "gaps" em seus léxicos, representações semânticas frágeis ou dificuldades em recuperar a informação na presença de representações bem elaboradas no léxico mental ${ }^{3}$.

Os campos conceituais Locais e Profissões foram os que apresentaram menor percentual de nomeação por Designações por Vocábulos Usuais (DVU), tanto no GDFN quanto no GDF. Menor expressão, para esses campos conceituais, foi também verificada em outros estudos $1,6,7,10$. A menor nomeação por Designações por Vocábulos Usuais (DVU), nesses campos conceituais pode ser justificada por fatores culturais ${ }^{10,28}$, do desenvolvimento ${ }^{28} \mathrm{e}$ também pelo input visual fornecido, visto que a nomeação de figuras envolve informações do tipo visual, semântica e fonológica ${ }^{1}$.

Relacionado à Não Designações (ND), o GDF apresentou maior média de percentual que o GDFN para todos os campos conceituais, havendo diferença estatisticamente significante para Vestuário, Alimentos, Meios de Transportes e Móveis e Utensílios. Corroborando esses achados, outros estudos ${ }^{9,10}$ mostraram que as primeiras 50 pala- vras adquiridas pelas crianças são, em sua grande maioria, nomes de pessoas, de animais, de alimentos e bebidas, de brinquedos e verbos. Além disso, as crianças com dificuldades lexicais podem ter decréscimo nas representações semânticas, com menor habilidade de nomeação de palavras ${ }^{4}$.

No que se refere aos Processos de Substituição (PS), por campo conceitual, a média do percentual do GDF foi maior que a média do GDFN, não havendo diferença estatística significante apenas para o campo conceitual Formas e Cores. Isso pode ser justificado pelo fato de que crianças com dificuldades no acesso às palavras estão mais dispostas a substituírem as palavras pretendidas (palavrasalvo) por aquelas mais frequentes e aprendidas mais cedo ${ }^{11}$. Estudo recente ${ }^{2}$ revela que quando o vocábulo esperado não é utilizado, entende-se que o termo mais apropriado ainda não foi adquirido.

Além disso, os achados desta pesquisa visam à importância de incluir a avaliação do vocabulário nos procedimentos de avaliação clínica de crianças com desvio fonológico, contribuindo com a prática fonoaudiológica através de uma avaliação clinica mais completa e de uma intervenção mais adequada e eficiente.

\section{CONCLUSÃO}

A partir da análise dos dados obtidos, o desempenho no vocabulário de crianças com desvio fonológico é inferior ao de crianças com desenvolvimento fonológico normal. Dessa forma, é importante realizar a avaliação do vocabulário nas crianças com desvio fonológico, a fim de detectar possível alteração no nível semântico e auxiliar no processo terapêutico. 


\begin{abstract}
Purpose: to compare the performance in vocabulary test of children with or without phonological disorder. Method: the study involved 150 children, 75 of the Phonological Disorder Group (PDG) and 75 of the Typical Phonological Developmental Group (TPDG), with age group ranging from 6:0 to 6:11 years, of both genders, with medium socioeconomic level. Auditory and speech-language screening were accomplished, and after that the children were submitted to the Child's Phonological Assessment and a Vocabulary Test. The answers were analyzed considering the number of Usual Verbal Designations (UVD), No Designations (ND) and Processes of Substitutions (PS) by conceptual field. Finally, data were tabulated and submitted to statistical analysis using the T-Test for independent samples and the significance level was considered 5\%. Results: PDG has obtained a smaller number of UVD that the TPDG, being difference statistically significant for all conceptual fields. Besides this, the occurrence of ND e PS were higher for PDG in all of conceptual fields, being the difference statistically significant for four and eight conceptual fields, respectively. Still, the occurrences of PS were larger than the ND for the two groups. Conclusion: the results indicated that PDG showed lower performance in the vocabulary test, and it were verified by the smaller occurrence of UVD and larger of ND and PS than the TPDG. These findings showed that have a relation between the phonological disorder and vocabulary deficit. So, it is important to evaluates the vocabulary of the children with phonological disorders in order to assist the therapy.
\end{abstract}

KEYWORDS: Vocabulary; Speech-Language Pathology; Semantics; Child

\section{REFERÊNCIAS}

1. Befi-Lopes DM, Gândara JP. Desempenho em prova de vocabulário de crianças com diagnóstico de alteração fonológica. Rev Soc Bras Fonoaudiol. 2002; 7(1):16-23.

2. Hage SRV, Pereira MB. Desempenho de crianças com desenvolvimento típico de linguagem em prova de vocabulário expressivo. Rev. CEFAC. 2006; 8(4):419-28. http://dx.doi.org/10.1590/ S1516-18462006000400003

3. McGregor KK, Friedman RM, Reilly RM, Newman RM. Semantic representation and naming in young children. J Speech Lang Hear Res. 2002; 45(2):33246. http://dx.doi.org/10.1044/1092-4388(2002/026)

4. Nash M, Donaldson ML. Word learning in children with vocabulary deficits. J Speech Lang Hear Res. 2005; 48(2):439-58. http://dx.doi. org/10.1044/1092-4388(2005/030)

5. Beitchman JH, Jiang H, Koyama E, Johnson CJ, Escobar M, Atkinson L, etal. Models and determinants of vocabulary growth from kindergarten to adulthood. J Child Psychol Psychiatr. 2008; 49(6):626-34. http://dx.doi.org/10.1111/j.1469-7610.2008.01878.x 6. Mota HB, Kaminski TI, Nepomuceno MRF, Athayde ML. Alterações no vocabulário expressivo de crianças com desvio fonológico. Rev Soc Bras Fonoaudiol. 2009; 14(1):41-7.

7. Athayde ML. Vocabulário expressivo e habilidades de memória de trabalho em crianças com desenvolvimento fonológico normal e desviante [dissertação] Santa Maria (RS): Universidade Federal de Santa Maria; 2009.

8. Gershkoff-Stowe L, Hahn ER. Fast mapping skills in the developing lexicon. J Speech Lang Hear Res. 2007; 50(3):682-97. http://dx.doi. org/10.1044/1092-4388(2007/048)

9. Bastos JC, Ramos APF, Marques J. Estudo do vocabulário infantil: limitações das metodologias tradicionais de coleta. Rev Soc Bras Fonoaudiol. 2004; 9:1-9.

10. Pedromônico MRM, Affonso LA, Sañudo A. Vocabulário expressivo de crianças entre 22 e 36 meses: estudo exploratório. Rev Bras Cresc Desenvolv Hum. 2002; 12(2):13-22.

11. German DJ, Newman RS. The impact of lexical factors on children's word-finding errors. J Speech Lang Hear Res. 2004; 47(3):624-36. http://dx.doi. org/10.1044/1092-4388(2004/048)

12. MesserD, DockrelJE.Children'snamingandwordfinding difficulties: descriptions and explanations. J Speech Lang Hear Res. 2006; 49(2):309-24. http:// dx.doi.org/10.1044/1092-4388(2006/025)

13. Scheuer $\mathrm{Cl}$, Stivanin L, Mangilli LD. Nomeação de figuras e a memória em crianças: efeitos fonológicos e semânticos. Pró-Fono. 2004; 16(1):49-56.

14. Maekawa J, Storkel HL. Individual differences in the influence of phonological characteristics 
on expressive vocabulary development by young children. J Child Lang. 2006; 33(3):439-59.

15. Hoff E, Naigles L. How children use input to acquire a lexicon. Child Dev. 2002; 73(2):418-33.

16. Basílio CS, Puccini RF, Silva EMK, Pedromônico MRM. Living conditions and receptive vocabulary of children aged two to five years. Rev Saúde Públ. 2005; 39(5):725-30. http://dx.doi.org/ S0034-89102005000500005

17. Hoff E, Core C, Bridges K. Non-word repetition assesses phonological memory and is related to vocabulary development in 20-to 24-month-olds. J Child Lang. 2008; 35(4):903-16. http://dx.doi. org/10.1017/S0305000908008751

18. Bishop DVM. Uncommon understanding, development and disorders of language comprehension in children. New York: Psychology Press; 2004. 293p.

19. Grunwell P. Os desvios fonológicos evolutivos numa perspectiva linguística. In: Yavas $\mathrm{M}$, organizador. Desvios fonológicos em crianças. Porto Alegre: Mercado Aberto; 1990. p.51-82.

20. Athayde ML, Carvalho Q, Mota HB. Vocabulário expressivo de crianças com diferentes níveis de gravidade de desvio fonológico. Rev. CEFAC. 2009; 11(Supl2):161-8. http://dx.doi.org/10.1590/ S1516-18462009000600005

21. Befi-Lopes MD. Verificação do vocabulário. In: Andrade CRF, Befi-Lopes DM, Fernandes FDM,
Wertzner HF. ABFW - Teste para avaliação da linguagem infantil - Parte B. São Paulo: Pró-Fono; 2000. p.41-60.

22. Fundação de Economia e Estatística (FEE) [homepage na internet] Porto Alegre: 2003. [acesso em dez 2008]. Disponível em: URL: http://www.fee. tche.br/sitefee/pt/content/estatisticas/pg_idese.php. 23. Yavas M, Hernandorena CM, Lamprecht RR. Avaliação fonológica da criança: reeducação e terapia. 2. ed. Porto Alegre: Artes Médicas; 2002. 24. Vieira MG, Mota HB, Keske-Soares M. Relação entre idade, grau de severidade do desvio fonológico e consciência fonológica. Rev Soc Bras Fonoaudiol. 2004; 9(3):144-50.

25. Mecca FDL. Investigação das funções da linguagem, da teoria da mente, do vocabulário e do desempenho escolar de alunos surdos. Rev Soc Bras Fonoaudiol. 2005; 10(4):250.

26. Tonietto L, Parente MAMP, Duvignau K, Gaume $B$, Bosa CA. Aquisição inicial do léxico verbal e aproximações semânticas em português. Psicol Reflex Crit. 2007; 20(1):114-23.

27. Johnson CJ, Paivio A, Clark JM. Cognitive components of picture naming. Psychol Bull. 1996; 120(1):113-39.

28. Miranda MC, Pompéia S, Bueno OFA. Um estudo comparativo das normas de um conjunto de 400 figuras entre crianças brasileiras e americanas. Rev Bras Psiquiatr. 2004; 26(4):226-33.

doi: 10.1590/S1516-18462010005000011

RECEBIDO EM: 21/08/2009

ACEITO EM: 20/12/2009

Endereço para correspondência:

Márcia Keske-Soares

RST 287, 900

Santa Maria - RS

CEP: 97060-500

E-mail: keske-soares@uol.com.br 